\title{
A Simple Dual Decomposition Method for Resource Allocation in Telecommunication Networks
}

\author{
Igor Konnov ${ }^{1, a}$, Aleksey Kashuba ${ }^{2}$ and Erkki Laitinen ${ }^{3}$ \\ ${ }^{1}$ Department of System Analysis and Information Technologies, Kazan Federal University, Kazan 420008, Russia \\ ${ }^{2}$ LLC "AST Povolzhye", Kazan 420029, Russia \\ ${ }^{3}$ Department of Mathematical Sciences, University of Oulu, 90014 Oulu, Finland
}

\begin{abstract}
We consider a problem of optimal resource allocation in a wireless communication network divided into zones (clusters). The network manager aims to distribute some homogeneous resource (bandwidth) among users of several zones in order to maximize the total network profit, which takes into account payments from users and implementation costs. As a result, we obtain a convex optimization problem involving capacity and balance constraints. By using the dual Lagrangian method with respect to the capacity constraint, we reduce the initial problem to a suitable one-dimensional problem, so that calculation of its cost function value leads to independent solution of zonal problems, treated as two-side auction models with one trader. We show that solution of each zonal problem can be found exactly by a simple arrangement type algorithm even in the case where the trader price is not fixed. Besides, we suggest ways to adjust the basic problem to the case of moving nodes. Some results of computational experiments confirm the applicability of the new method.
\end{abstract}

\section{Introduction}

The current development of telecommunication systems creates a number of new challenges of efficient management mechanisms for efficient allocation of limited communication networks resources. In fact, despite the existence of powerful processing and transmission devices, increasing demand of different communication services and its variability lead to serious congestion effects and inefficient utilization of network resources (e.g., bandwidth and batteries capacity), especially in wireless telecommunication networks. This situation forces one to replace the fixed allocation rules with more flexible mechanisms, which are based on proper mathematical models; see e.g. [1-3]. The problem is to suggest such models and to develop suitable solution methods. Usually, the decision making processes are based on solutions of the corresponding optimization problems. At the same time, experience of dealing with these very complicated and spatially distributed systems usually shows that these problems have to utilize a proper decomposition/clustering approach, which can be based on zonal, time, frequency and other attributes of nodes/units; see e.g. [4, 5].

In this paper, we consider one of such problems, i.e. optimal allocation of a homogeneous resource in a zonal telecommunication network such that the income received from users payments is maximized and the implementation costs of the network operator are minimized; see [6,7]. The network manager problem consists in optimal distribution of the resource shares

${ }^{\mathrm{a}}$ Corresponding author: konn-igor@yandex.ru among zones in order to maximize the total network profit. As a result, we obtain a convex optimization problem involving capacity and balance constraints. By using the dual Lagrangian method with respect to the capacity constraint, we reduce the initial problem to a suitable one-dimensional problem, so that calculation of its cost function value leads to independent solution of zonal problems, treated as two-side auction models with one trader. We show that solution of each zonal problem can be found exactly by a simple arrangement type algorithm even in the case where the trader price is not fixed. In such a way we develop a new dual decomposition approach for solution finding, whose implementation is simpler essentially in comparison with the methods from [6, 7]. We present results of computational experiments which confirm the applicability of the new method.

\section{Notation and the problem statement}

Let us consider a telecommunication network with nodes attributed to users (consumers) which is divided into zones (clusters). The problem of a manager of the network is to find the optimal allocation of a limited homogeneous network resource among the zones. That is, the optimal shares should maximize the value of the total profit containing the total income received from consumers' fees and negative resource implementation costs.

Let us use the following notation: 
- $n$ is the number of zones;

- $I_{k}$ is the index set of users (currently) located in zone $k(k=1, \ldots, n)$;

- $B$ is the total resource supply (the total bandwidth) for the system (network);

- $x_{k}$ is an unknown quantity of the resource allotted to zone $k$ with the upper bound $b_{k}$ and $f_{k}\left(x_{k}\right)$ is the cost of implementation of this quantity of the resource for zone $k(k=1, \ldots, n)$;

- $y_{i}$ is the resource amount received by user $j$ with the upper bound $a_{i}$ and $\varphi_{i}\left(y_{i}\right)$ is the charge value paid by user $j$ for the resource value $y_{i}$.

The problem of the network manager can be written as follows:

$$
\max \rightarrow \mu(x, y)=\sum_{k=1}^{n}\left[\sum_{i \in I_{k}} \varphi_{i}\left(y_{i}\right)-f_{k}\left(x_{k}\right)\right],
$$

subject to

$$
\begin{aligned}
& \sum_{i \in I_{k}} y_{i}=x_{k}, k=1, \ldots, n ; \\
& 0 \leq y_{i} \leq a_{i}, i \in I_{k}, k=1, \ldots, n ; \\
& \sum_{k=1}^{n} x_{k} \leq B ; \\
& 0 \leq x_{k} \leq b_{k}, k=1, \ldots, n .
\end{aligned}
$$

That is, (2) provides the balance for demand and supply in each zone, (3) and (5) are capacity constraints for users and network supply values in each zone, respectively, and (4) gives the upper bound for the total resource supply. The goal of the network manager is to maximize the total network profit subject to all these constraints.

In what follows we assume that there exists at least one feasible point satisfying conditions (2)-(5), each function $f_{k}\left(x_{k}\right)$ is convex and differentiable, and all the functions $\varphi_{i}\left(y_{i}\right)$ are affine, i.e.

$$
\varphi_{i}\left(y_{i}\right)=\alpha_{i} y_{i}+\beta_{i}, \alpha_{i}>0, i \in I_{k}, k=1, \ldots, n .
$$

This means that the prices (marginal utilities) $\alpha_{i}$ of the users are fixed, but the manager can vary the prices depending on volumes, so that each zonal price is a nonincreasing function.

\section{Dual solution method}

Under the basic assumptions of the previous section, (1)-(5) is a differentiable convex optimization problem, which has a solution since its feasible set is bounded. Hence it can be found by a great number of iterative methods; see e.g. [8, 9]. However, the problem of selection of an efficient decomposition method here is not trivial task since problem (1)-(5) has $n+1$ functional constraints (2) and (4) and many box type ones. For instance, utilization of the standard duality approach which is based on defining the Lagrangian function with respect to all the functional constraints leads to a nonsmooth dual convex optimization problem in $n+1$ dual variables, whose solution may cause certain difficulties. For this reason, we intend to apply a special dual method, which takes into account peculiarities of this problem and does not require hard implementation procedures.

Let us first define the Lagrange function of problem (1)-(4) as follows:

$$
L(x, y, \lambda)=\mu(x, y)-\lambda\left(\sum_{k=1}^{n} x_{k}-B\right),
$$

i.e. we insert only the term corresponding to the the upper bound constraint for the total resource supply (4) with the Lagrangian multiplier $\lambda$. At the same time, we keep the zonal balance constraints (2) as well as the capacity constraints (3) and (5).

Hence, we can write the one-dimensional dual problem:

$$
\min _{\lambda \geq 0} \rightarrow \psi(\lambda)
$$

where

$$
\begin{aligned}
\psi(\lambda)= & \sup _{(x, y) \in W} L(x, y, \lambda) \\
= & \sup _{(x, y) \in W} \sum_{k=1}^{n}\left[\sum_{i \in I_{k}} \varphi_{i}\left(y_{i}\right)-f_{k}\left(x_{k}\right)-\lambda x_{k}\right] \\
& +\lambda B ;
\end{aligned}
$$

where

$$
W=\left\{\begin{array}{l|l}
(x, y) & \begin{array}{l}
\sum_{i \in I_{k}} y_{i}=x_{k} \\
0 \leq y_{i} \leq a_{i}, i \in I_{k}, \\
0 \leq x_{k} \leq b_{k}, k=1, \ldots, n
\end{array}
\end{array}\right\}
$$

By duality (see e.g. [8, 9]), problems (1)-(5) and (7) have the same optimal value. However, solution of (7) can be found by one of well-known one-dimensional optimization algorithms based on calculation of values of $\psi(\lambda)$. We now discuss this problem in more detail. The main element in calculation of $\psi(\lambda)$ is a solution of the problem:

$$
\max \rightarrow \sum_{k=1}^{n}\left[\sum_{i \in I_{k}} \varphi_{i}\left(y_{i}\right)-f_{k}\left(x_{k}\right)-\lambda x_{k}\right]
$$

subject to

$$
\begin{aligned}
& \sum_{i \in I_{k}} y_{i}=x_{k}, 0 \leq y_{i} \leq a_{i}, i \in I_{k}, \\
& 0 \leq x_{k} \leq b_{k}, k=1, \ldots, n .
\end{aligned}
$$


However, this problem decomposes into $n$ independent zonal convex programming problems

$$
\max \rightarrow\left[\sum_{i \in I_{k}} \varphi_{i}\left(y_{i}\right)-f_{k}\left(x_{k}\right)-\lambda x_{k}\right]
$$

subject to

$$
\begin{aligned}
& \sum_{i \in I_{k}} y_{i}=x_{k}, 0 \leq y_{i} \leq a_{i}, i \in I_{k}, \\
& 0 \leq x_{k} \leq b_{k},
\end{aligned}
$$

for $k=1, \ldots, n$. Hence, we have to suggest a simple and efficient algorithm for the basic problem (9).

Set $y(k)=\left(y_{i}\right)_{i \in I_{k}}$,

$$
W_{k}=\left\{\begin{array}{l|l}
\left(x_{k}, y(k)\right) \mid \begin{array}{l}
\sum_{i \in I_{k}} y_{i}=x_{k}, \\
0 \leq y_{i} \leq a_{i}, i \in I_{k}, \\
0 \leq x_{k} \leq b_{k}
\end{array}
\end{array}\right\},
$$

then

$$
W=\prod_{k=1}^{n} W_{k} .
$$

The necessary and sufficient optimality condition for problem (9) in view of (6) is written in the form of the variational inequality: find $\left(\bar{x}_{k}, \bar{y}(k)\right) \in W_{k}$ such that

$$
\begin{aligned}
& \left(f_{k}^{\prime}\left(\bar{x}_{k}\right)+\lambda\right)\left(x_{k}-\bar{x}_{k}\right)-\sum_{i \in I_{k}} \alpha_{i}\left(y_{i}-\bar{y}_{i}\right) \geq 0 \\
& \forall\left(x_{k}, y(k)\right) \in W_{k} .
\end{aligned}
$$

This is a two-sided auction type equilibrium problem with one seller and several buyers; see e.g. $[10,11]$. It is equivalent to the problem of finding a feasible vector $\left(\bar{x}_{k}, \bar{y}(k)\right) \in W_{k}$ and a cutting price $\bar{p}_{k}$ such that

$$
f_{k}^{\prime}\left(\bar{x}_{k}\right)+\lambda\left\{\begin{array}{lll}
\geq \bar{p}_{k} & \text { if } & \bar{x}_{k}=0, \\
=\bar{p}_{k} & \text { if } & \bar{x}_{k} \in\left(0, b_{k}\right), \\
\leq \bar{p}_{k} & \text { if } & \bar{x}_{k}=b_{k},
\end{array}\right.
$$

and

$$
\alpha_{i}\left\{\begin{array}{lll}
\leq \bar{p}_{k} & \text { if } & \bar{y}_{i}=0, \\
=\bar{p}_{k} & \text { if } & \bar{y}_{j} \in\left(0, a_{i}\right), \quad i \in I_{k} . \\
\geq \bar{p}_{k} & \text { if } & \bar{y}_{j}=a_{i},
\end{array}\right.
$$

Since buyers' prices are fixed, we can re-arrange them to be non-increasing and then find easily an intersection point of the staircase-wise inverse common demand and offer price $f_{k}^{\prime}\left(x_{k}\right)+\lambda$ lines; see also [11]. Therefore, an exact solution of problem (10) or (9) (hence (8)) can be found explicitly by simple ordering type algorithms, although (9) contain a non-linear function in general. In other words, calculation of values of $\psi(\lambda)$ can be accomplished by several independent simple ordering type algorithms. Notice that the re-arrangement of bid prices $\alpha_{i}$ in each zone should be made only one time that reduces the computational expenses essentially in comparison with the general duality approach. So, having the optimal value $\lambda^{*}$ of problem (7), we can find a solution of problem (1)-(5) by solving problem (8) with $\lambda=\lambda^{*}$, i.e. it is accomplished within the main calculation process for (7).

\section{Adjustment for the case of moving nodes}

In the above model it was assumed that users locations are fixed. We now intend to suggest some adjustments of the above model to networks with more complex and non-stationary behavior of users (nodes), which is typical for various modern wireless telecommunication systems; see e.g. $[2,12]$.

We consider the above problem of the network manager for some time slot. In this case we need some additional information about the behavior of users (nodes). It was suggested by I. Konnov (see e.g. [13]) to treat each moving node in a wireless network as a separate Markovian chain.

In order to create such a model, we determine a suitable grid $G$ covering the domain of the network so that $\mathrm{G}_{k}$ denotes the index set of all the cells belonging to zone $k$. Next, we consider the discrete time model and suppose that, given a user (node) $j$, we can determine the starting probability vector $\pi^{j,(0)}$, whose components $\pi_{\sigma}^{j,(0)}$ give its probabilities to be in cell $\sigma \in \mathrm{G}$ by time slot (stage) 1 , and the probability $\tilde{\pi}_{\sigma \tau}^{j}$ (for the simplicity of exposition, it is supposed to be independent of time) of the one stage transition $\sigma \rightarrow \tau$ for each pair $\sigma, \tau \in \mathrm{G}$. Knowing the starting and transition probability vectors for each node $j$, we can calculate its probability $\pi_{\sigma}^{j,(t-1)}$ to be in cell $\sigma \in \mathrm{G}$ by a selected slot $t$ via the standard Markovian chain technique (see e.g. [14]). Afterwards we calculate the value

$$
\tilde{p}_{k}^{j,(t-1)}=\sum_{\sigma \in \in_{k}} \pi_{\sigma}^{j,(t-1)}
$$

for each zone $k$ and assign user $j$ to zone $l$ where the probability $\widetilde{p}_{l}^{j(t-1)}$ is maximal, i.e. then $j \in I_{l}$.

Therefore, we can solve the same problem (7)-(9) with this assignment and obtain the desired resource allocation for time slot $t$ in the case of moving nodes.

Similarly, if

$$
\lim _{m \rightarrow \infty}\left(\Pi^{j}\right)^{m}=\bar{\Pi}^{j}
$$

for each probability matrix $\widetilde{\Pi}^{j}=\left(\tilde{\pi}_{\sigma \tau}^{j}\right)_{\{\sigma, \tau \in G\}}$, behavior of each user is stable and we can evaluate the optimal 
resource allocation for a long-time stationary period by calculation of the limit probabilities

$$
\bar{\pi}_{\tau}^{j}=\sum_{\sigma \in \mathrm{G}} \pi_{\sigma}^{j,(0)} \bar{\pi}_{\sigma \tau}^{j} \text { for } \tau \in \mathrm{G}
$$

and set

$$
\bar{p}_{k}^{j}=\sum_{\sigma \in \mathrm{G}_{k}} \bar{\pi}_{\sigma}^{j}
$$

for each $j$. Then we can assign user $j$ to zone $l$ where the probability $\bar{p}_{l}^{j}$ is maximal, i.e. then $j \in I_{l}$ and solve problem (7)-(9) with this assignment and obtain the longtime resource allocation strategy.

However, this approach can not be used if the limit in (13) does not exist. Then we can apply the statistical approach and calculate the probabilities on-line as it was suggested in [15]. After $t$ time slots we can determine the value

$$
p_{k}^{j, t}=s_{j, k}(t) / t
$$

for each user $j$ and for each zone $k$, where $s_{j, k}(t)$ denotes the number of time slots when user $j$ was in zone $k$. It is treated as some approximation of the probability of user $j$ to be in zone $k$. We set

$$
\bar{p}_{k}^{j}=p_{k}^{j, t}
$$

if

$$
\left\{\sum_{j \in I} \sum_{k=1}^{n}\left(p_{k}^{j, t}-p_{k}^{j, t-1}\right)^{2}\right\}^{1 / 2} \leq \delta
$$

for $\delta>0$ small enough, where $I$ denotes the index set of all the users. Then we utilize the values $\bar{p}_{k}^{j}$ as above in order to assign each user $j$ to some zone $l$. Solution of problem (7)-(9) with this assignment gives the longtime resource allocation strategy.

\section{Numerical experiments}

In order to evaluate efficiency of the new method we made several series of computational experiments. Since the case of moving nodes yields the same mathematical model (1)-(5) we restricted ourselves with the fixed case.

We utilized the golden section method for solving the single-dimensional optimization problem (7). The programs were coded in $\mathrm{C}++$ with a $\mathrm{PC}$ with the following facilities: Intel(R) Core(TM) i7-4500, CPU $1.80 \mathrm{GHz}$, RAM $6 \mathrm{~Gb}$.

The initial intervals for choosing the dual variable $\lambda$ were taken as $[0,1000]$. Values of $b_{k}$ were chosen by trigonometric functions in $[1,11]$, values of $a_{i}$ were chosen by trigonometric functions in $[1,2]$. The functions $f_{k}\left(x_{k}\right)$ were chosen to be convex quadratic, all the coefficients of $f_{k}\left(x_{k}\right)$ and $\varphi_{i}\left(y_{i}\right)$ were chosen with the help of trigonometric functions. The number of zones was varied from 5 to 105 , the number of users was varied from 210 to 1010 . Users were distributed in zones either uniformly or according to the normal distribution. The processor time and number of iterations, which gave an approximate solution of problem (7) within the same accuracy, were not significantly different for these two cases of distributions. We made calculations with 1000 test examples for each set of parameters, their average values are indicated in each row of the tables.

Further we report the results of tests, which include the time and number of iterations needed to find a solution of problem (7) within some accuracies. Let $\varepsilon$ denote the desired accuracy of finding an approximate solution of problem (7). Let $J$ denote the total number of users, $N_{\varepsilon}$ the number of upper iterations in $\lambda, T_{\varepsilon}$ the total processor time in seconds. The results of computations are given in Tables 1-3. In Table 1, we vary the accuracy $\varepsilon$, in Tables 2 and 3 we vary the total number of users and the number of zones, respectively.

Table 1. Results of testing with $J=510, n=70$.

\begin{tabular}{|c|c|c|}
\hline$\varepsilon$ & $N_{\varepsilon}$ & $T_{\varepsilon}$ \\
\hline $10^{-1}$ & 20 & 0.0003 \\
\hline $10^{-2}$ & 24 & 0.0004 \\
\hline $10^{-3}$ & 29 & 0.0008 \\
\hline $10^{-4}$ & 34 & 0.0007 \\
\hline
\end{tabular}

Table 2. Results of testing with $n=70, \varepsilon=10^{-2}$.

\begin{tabular}{|c|c|c|}
\hline$J$ & $N_{\varepsilon}$ & $T_{\varepsilon}$ \\
\hline 210 & 24 & 0.0001 \\
\hline 310 & 24 & 0.0003 \\
\hline 410 & 24 & 0.0004 \\
\hline 510 & 24 & 0.0004 \\
\hline 610 & 24 & 0.0008 \\
\hline 710 & 24 & 0.0012 \\
\hline 810 & 24 & 0.0011 \\
\hline 910 & 24 & 0.0014 \\
\hline 1010 & 24 & 0.0018 \\
\hline
\end{tabular}

Table 3. Results of testing with $J=510, \varepsilon=10^{-2}$.

\begin{tabular}{|c|c|c|}
\hline$n$ & $N_{\varepsilon}$ & $T_{\varepsilon}$ \\
\hline 5 & 24 & 0.0001 \\
\hline 15 & 24 & 0.0002 \\
\hline 25 & 24 & 0.0001 \\
\hline 35 & 24 & 0.0001 \\
\hline 45 & 24 & 0.0002 \\
\hline 55 & 24 & 0.0002 \\
\hline 65 & 24 & 0.0002 \\
\hline 75 & 24 & 0.0002 \\
\hline 85 & 24 & 0.0003 \\
\hline 95 & 24 & 0.0004 \\
\hline 105 & 24 & 0.0002 \\
\hline
\end{tabular}


From the results we can conclude that the performance of the new method is satisfactory for applications.

\section{Conclusions}

Equations should be centred and should be numbered with the number on the right-hand side. In this work, we considered a problem of managing limited resources in a zonal wireless telecommunication network and gave its constrained convex optimization problem formulation. We proposed a new dual decomposition method, which reduces the initial problem to a sequence of simple zonal convex optimization problems, they can be solved by efficient ordering type algorithms despite the nonlinear cost network functions. The results of the numerical experiments confirmed the rapid convergence of these methods. We also suggested ways to adjust the problem to the case of moving nodes.

\section{References}

1. Courcoubetis, C., Weber, R.: Pricing Communication Networks: Economics, Technology and Modelling. John Wiley \& Sons, Chichester (2003)

2. Stańczak, S., Wiczanowski, M., Boche, H.: Resource Allocation in Wireless Networks. Theory and Algorithms. Springer, Berlin (2006)

3. Wyglinski, A.M., Nekovee, M., Hou, Y.T.: (eds.) Cognitive Radio Communications and Networks: Principles and Practice. Elsevier, Amsterdam (2010)

4. Chen, Y., Liestman, A.L.: A zonal algorithm for clustering ad hoc networks, Int. J. Found. of Computer Sci. 14, pp.305-322 (2003)

5. Rohloff, K., Ye, J., Loyall, J., Schantz, R.: A hierarchical control system for dynamic resource management, Proceedings of the 12th IEEE RealTime and Embedded Technology and Applications Symposium (RTAS 2006) Work in Progress Symposium, San Jose, CA (2006)

6. Konnov, I.V., Kashina, O.A., Laitinen, E.: Optimisation problems for control of distributed resources. Int. J. Model., Ident. and Contr., vol.14, pp.65-72 (2011)

7. Konnov, I.V., Kashina, O.A., Laitinen, E.: Two-level decomposition method for resource allocation in telecommunication network. Int. J. Dig. Inf. Wirel. Comm., vol.2, pp.150-155 (2012)

8. Polyak, B.T.: Introduction to Optimization. Nauka, Moscow, 1983 (Engl. transl. in Optimization Software, New York, 1987)

9. Minoux, M.: Programmation Mathématique. Théorie et Algorithmes. Bordas, Paris (1989)

10. Konnov, I.V.: On variational inequalities for auction market problems. Optim. Lett., vol.1, pp.155-162 (2007)

11. Konnov, I.V.: Optimisation of network resources with zonal auctions. Int. J. Knowl. Engin. Soft Data Parad., vol.4, pp.297-305 (2014)

12. X. Cheng, X. Huang, D.-Z. Du, Eds., Ad Hoc Wireless Networking, Kluwer, Dordrecht (2004)

13. I.V. Konnov, O. A. Kashina, Optimization based flow control in communication networks with moving nodes, Proc. of The Fourth Moscow Conference on Operations Research, MaxPress, Moscow, 2004, pp.116-118 (2004)

14. J.G. Kemeny, J.L. Snell, Finite Markov Chains, Van Nostrand, Princeton (1960)

15. I.V. Konnov, E. Laitinen, O. Pinyagina, Equilibrium based flow control in wireless networks with moving users, Proc. of the Finnish Operations Research Society 40th Anniversary Workshop (FORS40), Ed. by M.Collan, J.Hamalainen, P.Luukka. Lappeenranta, 2013, pp.30-33 (2013) 